\title{
The results of X-ray binary Cyg X-1 investigations based on the optical photometry and high-resolution spectroscopy
}

\author{
Eugenia A. Karitskaya \\ Astronomical Institute of RAS, 48 Pyatnitskaya str., Moscow 119017, Russia \\ email: karitsk@sai.msu.ru
}

\begin{abstract}
Selected results of 1994-2004 optical photometric and high-resolution spectral observations including those obtained in the frame of a coordinated CIS countries campaign "Optical Monitoring of Unique Astrophysical Objects" and their comparison with ASM/RXTE X-ray data are briefly reviewed.
\end{abstract}

Keywords. X-rays: binaries - black hole physics - accretion, accretion disks - stars: early-type stars: fundamental parameters (colors, luminosities, masses, radii, temperatures)

\section{Introduction}

Cyg X-1/HDE226868 is an X-ray binary system (the orbital period $P=5.6^{d}$ ) whose relativistic component is the first candidate to black hole. The optical component, an O9.7 Iab supergiant, is responsible for about $95 \%$ of the system optical luminosity. The remaining $5 \%$ are due to the accretion structure (disc and surrounding gas) near $\mathrm{BH}$. In spite of the fact that the investigations of $\mathrm{Cyg} \mathrm{X}-1$ are being carried on for almost 40 years and have resulted in about 1000 publications, a lot of phenomena in the system, its geometrical and physical parameters remain unclear.

\section{The main results of photometric researches}

In the frame of 1994-1998 international campaign "Optical Monitoring of Unique Astrophysical Objects" (Georgia, Kazakhstan, Russia, Uzbekistan, and Ukraine) 2258 Cyg X-1 UBVR observations were made during 407 nights. The main results are reported in papers Karitskaya, et al. (2000), Karitskaya, et al. (2001), Karitskaya (2002) and briefly described below. By comparing photoelectric (UBVR) and X-ray ASM/RXTE (3-12 $\mathrm{keV}$ ) flux variations we found different kinds of variability (orbital variations, different kinds of flares, dips, so-called precession period 147/294 days) and a correspondence between optical and X-ray variations. Cross-correlation analysis revealed lags between X-ray $(2-10 \mathrm{keV})$ and optical long-term variations equaling $7^{d}$ in 1996 and $12^{d}$ in $1997-$ 1998.

One item in our list of accretion instability evidence is the existence of several-day-long optical flares followed by X-ray ones coinciding with X-ray dips. X-ray flare lag lengths in respect to the optical ones are the same as for slow variations (see above). We proposed the scenario for such X-ray flares in which the matter flows in separate portions from the supergiant to the accretion structure.

The characteristic time of the matter transfer through the accretion disk (the time delay of X-ray responses) was about 7 days in Summer and Autumn 1996 and 12 days in 1997-98. These times are too short for $\alpha$ - disk model. We may suggest for example that the most accreting matter goes through a high latitude of the accretion structure. 


\section{The main results of high-resolution spectral researches}

We report the results of Cyg X-1 spectral monitoring over 2002-2004 (Karitskaya (2003), Karitskaya et al. (2005), Karitskaya, et al. (2006)). The observations were carried out with the echelle-spectrographs of the Peak Terskol Observatory (altitude $3100 \mathrm{~m}$, North Caucasus) $2 \mathrm{~m}$ telescope (70 spectra, spectral resolution $\mathrm{R}=13000$ or 45000 ) and BOAO (Korea) $1.8 \mathrm{~m}$ telescope ( 5 spectra, $R=30000$ ) covering most of the optical range. Signal-to-noise ratio is $\mathrm{S} / \mathrm{N}>100-200$ near $\mathrm{H}_{\alpha}$. The observations fell at different states of Cyg X-1 X-ray spectrum ("hard", "soft", and transitional). We used RXTE/ASM X-ray data. The spectra contain the supergiant absorption lines and emission components of $\mathrm{H}_{\alpha}$ and $\mathrm{HeII} \lambda 4686 \AA$ lines with complicated profiles showing variation with the orbital period $5.6^{d}$.

Optical spectral line profile variations were found during X-ray flare. The comparison of the observed and non-LTE model HI, HeI and MgII profiles is given. Tidal distortion of Cyg X-1 optical component and its illumination by X-ray emission are taken into account. We set limits on the optical component main characteristics $T_{\text {eff }}=30400 \pm 500 \mathrm{~K}$, $\log g=3.31 \pm 0.07$, and overabundance of $\mathrm{He}$ and $\mathrm{Mg}:[\mathrm{He} / \mathrm{H}]=0.43 \pm 0.06 \mathrm{dex},[\mathrm{Mg} / \mathrm{H}]=$ $0.4-0.6$ dex by using 2003-2004 spectra.

For Doppler tomography we used the method developed by Agafonov (2004), on the basis of the radioastronomical approach. The main features are deconvolution with introduction of a synthesized beam and the removal of distortions by sidelobes' impact on the summary image (after back projecting) using CLEAN algorithm. This method is developed specially for a small number of irregularly distributed observations. Tomography maps of Cyg X-1 using HeII $\lambda 4686 \AA$ profiles were constructed (Karitskaya et al. (2005)). A new method of parameter determination through Doppler tomograms was proposed and tested. The Doppler images and Roche lobe model allowed to put a limitation on the black hole / supergiant mass ratio $1 / 4<q<1 / 3$.

\section{Long-term supergiant variations in Cyg X-1 binary system}

The photometric and spectral variations point to the supergiant parameter changes on a time scale of tens of years. Line profile non-LTE simulations lead to the conclusion that the star radius has grown about 1-4\% from 1997 to 2003-2004 and the temperature decreased by 1300-2400 K (Karitskaya, et al. (2006)). This agrees with the X-ray activity growth in that period. The increasing of the degree of the Roche lobe filling go to intensification and instability of the matter outlet toward the X-ray source.

\section{Acknowledgements}

The work is supported by RFBR grants 04-02-16924 and 06-02-16234.

\section{References}

Agafonov, M. I. 2004, AN, 325, 259; and ibid., 325, 263

Karitskaya, E. A., Goranskij, V. P., Grankin, E. N. et al. 2000, Astron. Lett., 26, 22

Karitskaya, E. A., Voloshina, I. B., Goranskij, V. P., et al. 2001, Astron. Rep., 45, 350

Karitskaya, E. A. 2002, in: R. K. Manchanda \& B. Paul (eds.), Multi Colour Universe, Proc. Conf. TIFR, (Ebenezer Printing House: Mumbai), p. 45

Karitskaya, E. A. 2003, Kinematika i Fizika Nebesnykh Tel, Suppl., No. 4, 230

Karitskaya, E. A., Agafonov, M. I., Bochkarev, N. G. et al. 2005, Astron. Astrophys. Trans., 24, 383

Karitskaya, E. A., Lyuty, V. M., Bochkarev, N. G. et al. 2006, IBVS, No. 5678, 1 